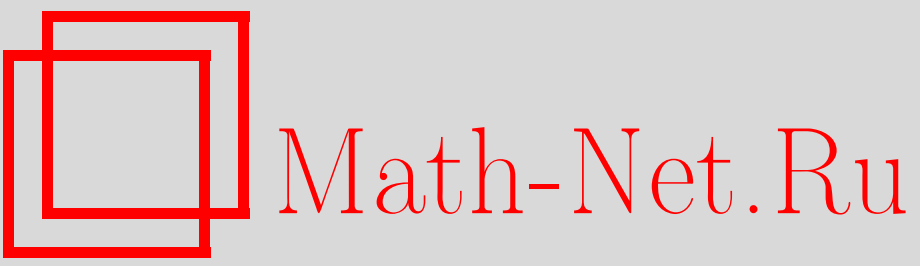

Ю. И. Скалько, С. Ю. Гриднев, Фундаментальное решение оператора задачи и его применение для приближенного решения начально-краевых задач, Итоги науки и техн. Сер. Соврем. мат. и ее прил. Темат. обз., 2021, том 193, 110-121

DOI: https://doi.org/10.36535/0233-6723-2021-193-110-121

Использование Общероссийского математического портала Math-Net.Ru подразумевает, что вы прочитали и согласны с пользовательским соглашением

http://www.mathnet.ru/rus/agreement

Параметры загрузки:

IP: 3.82 .47 .9

26 апреля 2023 г., 17:24:39 


\title{
ФУНДАМЕНТАЛЬНОЕ РЕШЕНИЕ ОПЕРАТОРА ЗАДАЧИ И ЕГО ПРИМЕНЕНИЕ ДЛЯ ПРИБЛИЖЕННОГО РЕШЕНИЯ НАЧАЛЬНО-КРАЕВЫХ ЗАДАЧ
}

\author{
(c) 2021 г. . И. СКАЛЬКО, С. Ю. ГРИДНЕВ
}

\begin{abstract}
АннотАция. В работе построено приближение фундаментального решения оператора задачи для гиперболической системы линейных дифференциальных уравнений первого порядка с постоянными коэффициентами. Предложен алгоритм приближенного решения обобщенной задачи Римана о распаде разрыва при наличии дополнительных условий на границах. Предложенный алгоритм сводит задачу нахождения значений переменных по обе стороны поверхности разрыва начальных данных к решению системы алгебраических уравнений с правой частью, зависящей от значений переменных в начальный момент времени в конечном числе точек. На основе этих решений построен вычислительный алгоритм приближенного решения начально-краевой задачи для гиперболической системы линейных дифференциальных уравнений первого порядка. Алгоритм реализован для системы уравнений упругой динамики и использован для решения некоторых прикладных задач, связанных с нефтедобычей.
\end{abstract}

Ключевые слова: распад разрыва, условия сопряжения, гиперболическая система, обобщенная функция, задача Коши, матрица-функция Грина, характеристика, инвариант Римана, уравнения упругой динамики.

FUNDAMENTAL SOLUTION OF AN OPERATOR

\section{AND ITS APPLICATION FOR THE APPROXIMATE SOLUTION OF INITIAL-BOUNDARY-VALUE PROBLEMS}

\author{
(c) 2021 YU. I. SKALKO, S. YU. GRIDNEV
}

\begin{abstract}
In this paper, we construct an approximation of the fundamental solution of a problem for a hyperbolic system of first-order linear differential equations with constant coefficients. We propose an algorithm for the approximate solution of the generalized Riemann problem on the discontinuity of a decay under additional conditions on the boundaries. This algorithm reduces the problem of finding values of variables on both sides of the discontinuity surface of the initial data to solving a system of algebraic equations whose right-hand sides depend on the values of the variables at the initial moment of time at a finite number of points. Based on these solutions, we develop a computational algorithm for the approximate solution of the initial-boundary-value problem for a hyperbolic system of firstorder linear differential equations. The algorithm is implemented for a system of equations of elastic dynamics; moreover, we use it to solve some applied problems related to oil production.
\end{abstract}

Keywords and phrases: decay of a discontinuity, conjugation conditions, hyperbolic system, generalized function, Cauchy problem, matrix Green function, characteristic, Riemann invariant, equations of elastic dynamics.

AMS Subject Classification: 35L40, 35L67, 35L45, 35L50 
1. Постановка задачи. Данная работа возникла из попыток решения конкретной прикладной задачи. Эта прикладная задача и обусловила особенности постановки математической задачи. Поэтому сначала несколько слов о прикладной задаче.

В многочисленных промышленных экспериментах на нефтеносных месторождениях убедительно показано, что установка на поверхности виброисточника и продолжительная его работа в течение нескольких месяцев ведет к существенному повышению нефтеотдачи нефтяного пласта. В ряде случаев эффект достигает 40\%. Механизмы и процессы, ведущие к такому повышению нефтеотдачи, остаются и на сегодня неясными. В частности, не понятно, каким образом энергия упругих волн, сгенерированных виброисточником, достигает значительных глубин (1 километр и более), избегая существенного рассеяния. Виброисточник с характерной мощностью 30 кВт и пятном контакта с породой порядка $1 \mathrm{~m}^{2}$ генерирует упругую волну. Если порода однородная, то энергия виброисточника рассеивается по полусфере и на глубинах порядка 1 км плотность энергии упругих волн убывает в $10^{6}$ раз даже при отсутствии поглощения в породе. Ожидать, что упругая волна такой малой плотности энергии вызовет какие-то значимые процессы в нефтеносном пласте, не приходится. Поскольку эффект увеличения нефтеотдачи в результате продолжительной работы поверхностного виброисточника многократно зафиксирован, то следует предположить, что при определенных условиях упругие волны распространяются в геологической породе, избегая существенного рассеяния. Мы попытались методами математического моделирования исследовать вопрос, может ли наличие трещин в геологической породе, в которых соприкасающиеся части могут смещаться друг относительно друга, приводить к тому, что упругая волна, сгенерированная виброисточником, не рассеивается по полусфере, а распространяется достаточно узким пучком так, что и на существенных глубинах ее плотность энергии остается значимой, чтобы вызвать те или иные физико-химические процессы.

Состояние геологической породы, в которой распространяется упругая волна, описывается векторным полем скорости смещений и тензором напряжений. Поскольку на трещине может происходить смещение частей породы друг относительно друга, то следовательно, при переходе через границу компоненты вектора скорости смещений и тензора напряжений могут претерпевать разрывы. Конечно, эти разрывы не могут быть произвольными, а обязаны удовлетворять определенным условиям, отражающим физические условия на границе соприкасающихся сред. Поскольку части геологической породы могут смещаться друг относительно друга только вдоль трещины, то компоненты вектора скорости смещений, направленные вдоль нормали к границе должны быть непрерывными при переходе через границу. Также в силу третьего закона Ньютона сила, действующая со стороны одной части породы на другую, равна и противоположно направлена силе, действующей со стороны второй части породы на первую. То есть нормальные к границе компоненты тензора напряжений должны быть равны по обе стороны границы. Если проскальзывание происходит без трения, то тангенциальные компоненты тензора напряжений должны быть равны нулю по обе стороны границы.

С другой стороны, всюду, кроме трещины, разделяющей части породы, геологическая порода является сплошной. Следовательно вектор смещений должен быть всюду (кроме трещины) непрерывным. Также требование выполнения третьего закона Ньютона в любом сечении ведет к непрерывности тензора напряжений. То есть всюду, кроме трещины, и вектор смещений и тензор напряжений непрерывны.

Исходя из сказанного, рассмотрим задачу: необходимо найти решение задачи Коши для системы линейных дифференциальных уравнений первого порядка с постоянными коэффициентами:

$$
\frac{\partial \boldsymbol{u}(t, \boldsymbol{x})}{\partial t}+\sum_{i=1}^{N} \boldsymbol{A}_{i} \frac{\partial \boldsymbol{u}(t, \boldsymbol{x})}{\partial x_{i}}=\mathbf{0}, \quad \boldsymbol{x} \in \mathbb{R}^{N}
$$

с начальными данными

$$
\boldsymbol{u}(t=0, \boldsymbol{x})=\boldsymbol{u}_{0}(\boldsymbol{x})
$$

которые всюду непрерывны, кроме гиперплоскости $\Gamma: x_{1}=0$. Решение должно быть всюду непрерывным, кроме гиперплоскости Г. Также должны выполняться заданные соотношения (условия 
сопряжения), связывающие значения переменных по обе стороны гиперплоскости $Г$ :

$$
\boldsymbol{L u}\left(t, x_{1}=-0, x_{2}, \ldots, x_{N}\right)+\boldsymbol{P} \boldsymbol{u}\left(t, x_{1}=+0, x_{2}, \ldots, x_{N}\right)=\mathbf{0} .
$$

Сформулированную постановку будем, следуя [5], называть обобщенной задачей Римана о распаде разрыва с условиями сопряжения на границах.

В случае одной пространственной переменной различными авторами [5-8] предложен ряд методов решения задачи Римана. По сути, все эти методы связаны с наличием у гиперболических систем характеристик. В случае многих пространственных переменных методы, основанные на наличии характеристик, уже не работают. И задача Римана чаще всего решается в предположении, что вблизи разрыва решение представляет собой плоскую волну, движущуюся вдоль нормали к поверхности разрыва $[5,8,9]$. Понятно, что такой подход далеко не во всех случаях является обоснованным.

В работе сформулирована постановка обобщенной задачи Римана о распаде разрыва с условиями сопряжения на границах для гиперболических систем линейных дифференциальных уравнений первого порядка с произвольным количеством пространственных переменных и приведен алгоритм построения ее решения. Этот алгоритм основан на нахождении фундаментального решения оператора задачи. Построенное решение обобщенной задачи Римана ляжет в основу вычислительного алгоритма нахождения приближенного решения начально-краевой задачи для описанного класса систем дифференциальных уравнений.

2. Обобщенные вектор-функции. В дальнейшем изложении будут использоваться понятия и утверждения теории обобщенных функций, изложение которой можно найти, например, в [2-4] или [1].

Определим пространство основных вектор-функций $\boldsymbol{S}\left(\mathbb{R}^{N}\right)$. Элементами этого пространства будут $M$-мерные вектор-функции $\boldsymbol{\varphi}=\left(\varphi_{1}, \ldots, \varphi_{M}\right)$, компоненты которых $\varphi_{1}(\boldsymbol{y}), \ldots, \varphi_{M}(\boldsymbol{y})$ принадлежат пространству $S\left(\mathbb{R}^{N}\right)$, которое состоит из функций класса $C^{\infty}\left(\mathbb{R}^{N}\right)$, убывающих при $|y| \rightarrow \infty$ вместе со всеми своими производными быстрее любой степени $|y|^{-1}$.

Определение 1. Обобщенными вектор-функциями $\boldsymbol{f}=\left(f_{1}, \ldots, f_{M}\right) \in \boldsymbol{S}^{\prime}\left(\mathbb{R}^{N}\right)$ будем называть линейные непрерывные функционалы на векторном основном пространстве $\boldsymbol{S}\left(\mathbb{R}^{N}\right)$. При этом функционал $\boldsymbol{f}$ действует на основную вектор-функцию $\boldsymbol{\varphi}=\left(\varphi_{1}, \ldots, \varphi_{M}\right)$ по формуле $(\boldsymbol{f}, \boldsymbol{\varphi})=$ $\left(f_{1}, \varphi_{1}\right)+\ldots+\left(f_{M}, \varphi_{M}\right)$.

Определение 2. Обобщенным решением для системы уравнений

$$
\frac{\partial \boldsymbol{u}(t, \boldsymbol{x})}{\partial t}+\sum_{i=1}^{N} \boldsymbol{A}_{i} \frac{\partial \boldsymbol{u}(t, \boldsymbol{x})}{\partial x_{i}}=\boldsymbol{f}(t, \boldsymbol{x})
$$

будем называть обобщенную функцию $\boldsymbol{u}(t, \boldsymbol{x}) \in \boldsymbol{S}^{\prime}\left(\mathbb{R}^{N+1}\right)$, удовлетворяющую этому уравнению в обобщенном смысле, т. е. для произвольной основной функции $\boldsymbol{\varphi}(t, \boldsymbol{x}) \in \boldsymbol{S}\left(\mathbb{R}^{N+1}\right)$ выполняется равенство

$$
\left(\frac{\partial \boldsymbol{u}}{\partial t}, \boldsymbol{\varphi}\right)+\sum_{i=1}^{N}\left(\boldsymbol{A}_{i} \frac{\partial \boldsymbol{u}}{\partial x_{i}}, \boldsymbol{\varphi}\right)=(\boldsymbol{f}, \boldsymbol{\varphi})
$$

$\boldsymbol{A}_{i}$ - матрицы коэффициентов системы уравнений (4), размера $(M \times M)$.

В дальнейшем будем полагать, что каждая из матриц $\boldsymbol{A}_{i}$ имеет полный набор левых собственных векторов и, следовательно, представима в виде

$$
\boldsymbol{A}_{i}=\boldsymbol{R}_{i} \boldsymbol{\Lambda}_{i} \boldsymbol{\Omega}_{i},
$$

$\boldsymbol{\Lambda}_{i}$ - диагональная матрица собственных чисел матрицы $\boldsymbol{A}_{i}$, упорядоченных по не убыванию, $\boldsymbol{\Omega}_{i}$ - матрица, строки которой являются левыми собственными векторами матрицы $\boldsymbol{A}_{i}$, соответствующие собственным числам $\boldsymbol{\Lambda}_{i}, \boldsymbol{R}_{i}=\boldsymbol{\Omega}_{i}^{-1}$ - матрица, столбцы которой являются правыми собственными векторами матрицы $\boldsymbol{A}_{i}$. 
Определение 3. Фундаментальным решением оператора задачи (4) или матрицей-функцией Грина называется обобщенная матрица-функция $\boldsymbol{G}(t, \boldsymbol{x}) \in \boldsymbol{S}^{\prime}\left(\mathbb{R}^{N+1}\right)$, удовлетворяющая уравнению

$$
\frac{\partial \boldsymbol{G}}{\partial t}+\sum_{i=1}^{N} \boldsymbol{A}_{i} \frac{\partial \boldsymbol{G}}{\partial x_{i}}=\boldsymbol{I} \delta(t, \boldsymbol{x}),
$$

где $\boldsymbol{I}$ - единичная диагональная матрица $(M \times M)$.

Определение 4. Сверткой $\boldsymbol{G} * \boldsymbol{f}$ обобщенной матрицы-функции $\boldsymbol{G}=G_{i j} \in \boldsymbol{S}^{\prime}$ и обобщенной вектор-функции $\boldsymbol{f}=f_{j} \in \boldsymbol{S}^{\prime}$ будем называть такую обобщенную вектор-функцию $\boldsymbol{u}=u_{i} \in \boldsymbol{S}^{\prime}$, что

$$
u_{i}=\sum_{j=1}^{M} G_{i, j} * f_{j},
$$

где $G_{i, j} * f_{j}$-свертка $G_{i, j}$ и $f_{j}$, рассматриваемых как обобщенные функции из $S^{\prime}$.

Сформулируем несколько лемм, доказательство которых приведено в [6].

Лемма 1. Пусть $\boldsymbol{f}(t, \boldsymbol{x}) \in \boldsymbol{S}^{\prime}$ таково, что свертка $\boldsymbol{G} * \boldsymbol{f}$ существует в $\boldsymbol{S}^{\prime}$. Тогда решение уравнения (4) существует в $\boldsymbol{S}^{\prime}$ и дается формулой

$$
\boldsymbol{u}=\boldsymbol{G} * \boldsymbol{f} .
$$

Это решение единственно в классе тех функций из $\boldsymbol{S}^{\prime}$, для которых существует свертка с $\boldsymbol{G}$.

Лемма 2. Пусть и $\boldsymbol{x})$-локально интегрируемая функция в $\mathbb{R}^{N}$. Тогда

$$
\theta(t) \delta(\boldsymbol{x}-\boldsymbol{a} t) * u(\boldsymbol{x}) \delta(t)=\theta(t) u(\boldsymbol{x}-\boldsymbol{a} t)
$$

Замечание 1. Как следует из доказанной леммы, значение свертки $\theta(t) \delta(\boldsymbol{x}-\boldsymbol{a t}) * u(\boldsymbol{x}) \delta(t)$ в точке $(\boldsymbol{x}, t)$ равно значению функции $u(\boldsymbol{x})$ в точке пересечения прямой $d \boldsymbol{x} / d t=\boldsymbol{a}$, проведенной через точку $(\boldsymbol{x}, t)$, с гиперплоскостью $t=0$.

Лемма 3. Пусть $v(t, \boldsymbol{x})$-локально интегрируемая функция в $\mathbb{R}^{N+1} u v(t, \boldsymbol{x})=0$ при $t \leqslant 0$, тогда если $a_{1} \neq 0$, то

$$
\theta(t) \delta(\boldsymbol{x}-\boldsymbol{a} t) * v(t, \boldsymbol{x}) \delta\left(x_{1}\right)=\frac{1}{\left|a_{1}\right|} \theta\left(\frac{x_{1}}{a_{1}}\right) v\left(t-\frac{x_{1}}{a_{1}}, \boldsymbol{x}-\frac{x_{1}}{a_{1}} \boldsymbol{a}\right),
$$

если $a_{1}=0$, mo

$$
\theta(t) \delta(\boldsymbol{x}-\boldsymbol{a} t) * v(t, \boldsymbol{x}) \delta\left(x_{1}\right)=0 .
$$

Замечание 2. Проведем через точку $(t>0, \boldsymbol{x})$ прямую линию $d \boldsymbol{x} / d t=\boldsymbol{a}$. Эта прямая пересекает гиперплоскость $x_{1}=0$ в момент времени $t^{*}=t-x_{1} / a_{1}$. Если этот момент времени лежит вне интервала $0 \leqslant t^{*} \leqslant t$, то $\theta(t) \delta(\boldsymbol{x}-\boldsymbol{a} t) * \theta(t) v(t, \boldsymbol{x}) \delta\left(x_{1}\right)=0$ в точке $(t>0, \boldsymbol{x})$.

3. Фундаментальное решение. Построим фундаментальное решение оператора задачи (4). Обозначим через $\boldsymbol{V}(t, \boldsymbol{\xi})=F_{\boldsymbol{x}}[\boldsymbol{G}]$ - преобразование Фурье $\boldsymbol{G}(t, \boldsymbol{x})$ по пространственным переменным. Выполним преобразование Фурье уравнений (6) по пространственным переменным. Учитывая, что $F_{\boldsymbol{x}}[\boldsymbol{G}]=-i \xi_{j} F_{\boldsymbol{x}}[\boldsymbol{G}]$, для обобщенной функции $\boldsymbol{V}(t, \boldsymbol{\xi})$ получаем уравнение

$$
\frac{\partial \boldsymbol{V}}{\partial t}-i \sum_{j=1}^{N} \xi_{j} \boldsymbol{A}_{j} \boldsymbol{V}=\boldsymbol{I} \delta(t) .
$$

Решение уравнения (8) имеет вид

$$
\boldsymbol{V}(t, \boldsymbol{\xi})=\theta(t) \exp \left(i \sum_{j=1}^{N} \xi_{j} \boldsymbol{A}_{j} t\right)
$$


где $\theta(t)$ - функция Хевисайда:

$$
\theta(t)= \begin{cases}1, & \text { если } x>0 \\ 0, & \text { если } x \leqslant 0 .\end{cases}
$$

По определению матричной экспоненты

$$
\exp \left(i \sum_{j=1}^{N} \xi_{j} \boldsymbol{A}_{j} t\right)=\prod_{j=1}^{N} \exp \left(i \xi_{j} \boldsymbol{A}_{j} t\right)+\sum_{|\boldsymbol{\alpha}| \geqslant 2} t^{|\boldsymbol{\alpha}|} \boldsymbol{B}_{\boldsymbol{\alpha}} \prod_{j=1}^{N}\left(-i \xi_{j}\right)^{\alpha_{j}} .
$$

Здесь $\boldsymbol{\alpha}=\left(\alpha_{1}, \alpha_{2}, \ldots, \alpha_{N}\right)$ - целочисленный вектор с неотрицательными составляющими $\alpha_{j}$ (мультииндекс), $|\boldsymbol{\alpha}|=\left(\alpha_{1}+\ldots+\alpha_{N}\right), \boldsymbol{B}_{\boldsymbol{\alpha}}$ - матрицы размера $M \times M$, являющиеся полиномами матриц $\boldsymbol{A}_{j}$ степени $|\boldsymbol{\alpha}|$. Учтем (5); тогда

$$
\exp \left(i \xi_{j} \boldsymbol{A}_{j} t\right)=\boldsymbol{R}_{j} \exp \left(i \xi_{j} \boldsymbol{\Lambda}_{j} t\right) \boldsymbol{\Omega}_{j}
$$

Следовательно,

$$
\exp \left(i \sum_{j=1}^{N} \xi_{j} \boldsymbol{A}_{j} t\right)=\prod_{j=1}^{N} \boldsymbol{R}_{j} \exp \left(i \xi_{j} \boldsymbol{\Lambda}_{j} t\right) \boldsymbol{\Omega}_{j}+\sum_{|\boldsymbol{\alpha}| \geqslant 2} t^{|\boldsymbol{\alpha}|} \boldsymbol{B}_{\boldsymbol{\alpha}} \prod_{j=1}^{N}\left(-i \xi_{j}\right)^{\alpha_{j}}
$$

Выполняя обратное преобразование Фурье, получим матрицу-функцию Грина

$$
\boldsymbol{G}(t, \boldsymbol{x})=\theta(t)\left(\prod_{j=1}^{N} \boldsymbol{R}_{j} \delta\left(\boldsymbol{I} x_{j}-\boldsymbol{\Lambda}_{j} t\right) \boldsymbol{\Omega}_{j}+\sum_{|\boldsymbol{\alpha}| \geqslant 2} t^{|\boldsymbol{\alpha}|} \boldsymbol{B}_{\boldsymbol{\alpha}} D^{\boldsymbol{\alpha}} \delta(\boldsymbol{x})\right) .
$$

Здесь $\delta\left(\boldsymbol{I} x_{j}-\boldsymbol{\Lambda}_{j} t\right)$ - диагональные матрицы, в $k$-й строке которых стоит обобщенная функция $\delta\left(x_{j}-\lambda_{j}^{k} t\right), \lambda_{j}^{k}-k$-е собственное число матрицы $\boldsymbol{A}_{j}$,

$$
D^{\boldsymbol{\alpha}}=\frac{\partial^{|\boldsymbol{\alpha}|}}{\partial x_{1}^{\alpha_{1}} \partial x_{2}^{\alpha_{2}} \ldots \partial x_{N}^{\alpha_{N}}}
$$

- оператор дифференцирования по пространственным переменным.

Рассмотрим сомножитель $\boldsymbol{R}_{j} \delta\left(\boldsymbol{I} x_{j}-\boldsymbol{\Lambda}_{j} t\right) \boldsymbol{\Omega}_{j}$. Обозначим через $\boldsymbol{D}^{k}$ квадратную матрицу размера $M \times M$, все элементы которой равны 0 , кроме $k$-го элемента главной диагонали, равного 1 . Тогда

$$
\boldsymbol{R}_{j} \delta\left(\boldsymbol{I} x_{j}-\boldsymbol{\Lambda}_{j} t\right) \boldsymbol{\Omega}_{j}=\sum_{k=1}^{M} \boldsymbol{R}_{j} \boldsymbol{D}^{k} \boldsymbol{\Omega}_{j} \delta\left(x_{j}-\lambda_{j}^{k} t\right)=\sum_{k=1}^{M} \boldsymbol{C}_{j}^{k} \delta\left(x_{j}-\lambda_{j}^{k} t\right) .
$$

Следовательно,

$$
\prod_{j=1}^{N} \boldsymbol{R}_{j} \delta\left(\boldsymbol{I} x_{j}-\boldsymbol{\Lambda}_{j} t\right) \boldsymbol{\Omega}_{j}=\sum_{k_{1}=1}^{M} \sum_{k_{2}=1}^{M} \ldots \sum_{k_{N}=1}^{M} \boldsymbol{C}_{1}^{k_{1}} \boldsymbol{C}_{2}^{k_{2}} \ldots \boldsymbol{C}_{N}^{k_{N}} \delta\left(x_{1}-\lambda_{1}^{k_{1}} t\right) \delta\left(x_{2}-\lambda_{2}^{k_{2}} t\right) \ldots \delta\left(x_{N}-\lambda_{N}^{k_{N}} t\right) .
$$

Если ввести мультииндекс $\boldsymbol{k}=\left(k_{1}, k_{2}, \ldots, k_{N}\right)$, т.е. целочисленный вектор с составляющими $k_{j}=1, \ldots, M$, многоиндексный массив матриц $\boldsymbol{C}^{\boldsymbol{k}}=\boldsymbol{C}_{1}^{k_{1}} \boldsymbol{C}_{2}^{k_{2}} \ldots \boldsymbol{C}_{N}^{k_{N}}$, многоиндексный массив векторов $\boldsymbol{\lambda}^{\boldsymbol{k}}=\left(\lambda_{1}^{k_{1}}, \lambda_{2}^{k_{2}}, \ldots, \lambda_{N}^{k_{N}}\right)$, то

$$
\prod_{j=1}^{N} \boldsymbol{R}_{j} \delta\left(\boldsymbol{I} x_{j}-\boldsymbol{\Lambda}_{j} t\right) \boldsymbol{\Omega}_{j}=\sum_{\boldsymbol{k}} \boldsymbol{C}^{\boldsymbol{k}} \delta\left(\boldsymbol{x}-\boldsymbol{\lambda}^{\boldsymbol{k}} t\right)
$$

Тогда

$$
\boldsymbol{G}(t, \boldsymbol{x})=\theta(t) \sum_{\boldsymbol{k}} \boldsymbol{C}^{\boldsymbol{k}} \delta\left(\boldsymbol{x}-\boldsymbol{\lambda}^{\boldsymbol{k}} t\right)+\boldsymbol{O}\left(t^{2}\right) .
$$

В случае двух пространственных переменых

$$
\boldsymbol{G}(t, \boldsymbol{x})=\theta(t) \sum_{\boldsymbol{k}} \boldsymbol{C}_{1}^{k_{1}} \boldsymbol{C}_{2}^{k_{2}} \delta\left(\boldsymbol{x}-\boldsymbol{\lambda}^{\boldsymbol{k}} t\right)+\frac{\theta(t)}{2} t^{2}\left(\boldsymbol{A}_{2} \boldsymbol{A}_{1}-\boldsymbol{A}_{1} \boldsymbol{A}_{2}\right) \frac{\partial^{2} \delta(\boldsymbol{x})}{\partial x_{1} \partial x_{2}}+\boldsymbol{O}\left(t^{3}\right) .
$$


Если поменять нумерацию пространственных переменных, то можем записать

$$
\boldsymbol{G}(t, \boldsymbol{x})=\theta(t) \sum_{\boldsymbol{k}} \boldsymbol{C}_{2}^{k_{2}} \boldsymbol{C}_{1}^{k_{1}} \delta\left(\boldsymbol{x}-\boldsymbol{\lambda}^{\boldsymbol{k}} t\right)-\frac{\theta(t)}{2} t^{2}\left(\boldsymbol{A}_{2} \boldsymbol{A}_{1}-\boldsymbol{A}_{1} \boldsymbol{A}_{2}\right) \frac{\partial^{2} \delta(\boldsymbol{x})}{\partial x_{1} \partial x_{2}}+\boldsymbol{O}\left(t^{3}\right) .
$$

Сопоставляя (9) и (10), получаем

$$
\boldsymbol{G}(t, \boldsymbol{x})=\theta(t) \sum_{\boldsymbol{k}} \overline{\boldsymbol{C}}^{\boldsymbol{k}} \delta\left(\boldsymbol{x}-\boldsymbol{\lambda}^{\boldsymbol{k}} t\right)+\boldsymbol{O}\left(t^{3}\right)
$$

здесь использовано обозначение $\overline{\boldsymbol{C}}^{\boldsymbol{k}}=\left(\boldsymbol{C}_{1}^{k_{1}} \boldsymbol{C}_{2}^{k_{2}}+\boldsymbol{C}_{2}^{k_{2}} \boldsymbol{C}_{1}^{k_{1}}\right) / 2$.

Обратим внимание на следующий факт, который будет использован в дальнейшем. Поскольку $\boldsymbol{C}^{k_{j}}=\boldsymbol{R}_{j} \boldsymbol{D}^{k_{j}} \boldsymbol{\Omega}_{j}$, то

$$
\sum_{k_{j}=1}^{M} \boldsymbol{C}^{k_{j}}=\boldsymbol{R}_{j}\left(\sum_{k_{j}=1}^{M} \boldsymbol{D}^{k_{j}}\right) \boldsymbol{\Omega}_{j}=\boldsymbol{R}_{j} \boldsymbol{I} \boldsymbol{\Omega}_{j}=\boldsymbol{I} .
$$

4. Задача Римана. Пусть $\boldsymbol{u}(t, \boldsymbol{x})$ - решение задачи Римана (1), (2), (3). Введем обозначение

$$
\mathbf{y v}(t, \boldsymbol{x})=\theta(t)\left(\boldsymbol{u}\left(t, x_{1}=+0, x_{2}, \ldots, x_{N}\right)-\boldsymbol{u}\left(t, x_{1}=-0, x_{2}, \ldots, x_{N}\right)\right) .
$$

Покажем, что $\boldsymbol{u}(t, \boldsymbol{x})$, рассматриваемая как обобщенная функция из $\boldsymbol{S}^{\prime}$, удовлетворяет уравнению

$$
\frac{\partial \boldsymbol{u}}{\partial t}+\sum_{i=1}^{N} \boldsymbol{A}_{i} \frac{\partial \boldsymbol{u}}{\partial x_{i}}=\boldsymbol{u}_{0} \delta(t)+\boldsymbol{A}_{1} \mathbf{y} \mathbf{v} \delta\left(x_{1}\right) .
$$

Действительно, при всех $\boldsymbol{\varphi}(t, \boldsymbol{x}) \in \boldsymbol{S}$ имеем цепочку равенств:

$$
\begin{gathered}
\left(\frac{\partial \boldsymbol{u}}{\partial t}+\sum_{i=1}^{N} \boldsymbol{A}_{i} \frac{\partial \boldsymbol{u}}{\partial x_{i}}, \boldsymbol{\varphi}\right)=-\int\left(\frac{\partial \varphi^{T}}{\partial t} \boldsymbol{u}+\sum_{i=1}^{N} \frac{\partial \varphi^{T}}{\partial x_{i}} \boldsymbol{A}_{i} \boldsymbol{u}\right) d t d \boldsymbol{x}=\ldots \\
\ldots=\int\left(\boldsymbol{\varphi}^{T}\left(\frac{\partial \boldsymbol{u}}{\partial t}+\sum_{i=1}^{N} \boldsymbol{A}_{i} \frac{\partial \boldsymbol{u}}{\partial x_{i}}\right)\right) d t d \boldsymbol{x}+\int\left(\varphi^{T}(0, \boldsymbol{x}) \boldsymbol{u}(0, \boldsymbol{x})\right) d \boldsymbol{x}+\int_{\Gamma}\left(\boldsymbol{\varphi}^{T} \boldsymbol{A}_{1} \mathbf{y} \mathbf{v}\right) d t d \Gamma
\end{gathered}
$$

откуда и вытекает равенство (12).

Решение уравнения (12) представимо в виде свертки

$$
\boldsymbol{u}=\boldsymbol{G} * \boldsymbol{u}_{0} \delta(t)+\boldsymbol{G} * \boldsymbol{A}_{1} \mathbf{y} \mathbf{v} \delta\left(x_{1}\right) .
$$

На основании лемм 2 и 3 для точек $\boldsymbol{x}=\left(x_{1} \leqslant 0, x_{2}, \ldots, x_{N}\right)$, лежащих в левой полуплоскости, с точностью до $\boldsymbol{O}\left(t^{2}\right)$ можем записать

$$
\boldsymbol{u}\left(t, x_{1} \leqslant 0, x_{2}, \ldots, x_{N}\right)=\sum_{\boldsymbol{k}} \boldsymbol{C}^{\boldsymbol{k}} \boldsymbol{u}_{0}\left(\boldsymbol{x}-\boldsymbol{\lambda}^{\boldsymbol{k}} t\right)-\sum_{\boldsymbol{k}: x_{1}>\lambda_{-}^{k_{1}} t} \frac{\boldsymbol{C}^{\boldsymbol{k}} \boldsymbol{A}_{1}}{\lambda^{k_{1}}} \mathbf{y} \mathbf{v}\left(t-\frac{x_{1}}{\lambda^{k_{1}}}, \boldsymbol{x}-\frac{x_{1}}{\lambda^{k_{1}}} \boldsymbol{\lambda}^{\boldsymbol{k}}\right) .
$$

Переходя в $(13)$ к пределу $x_{1} \rightarrow-0$, получаем

$$
\boldsymbol{u}\left(t, x_{1}=-0, x_{2}, \ldots, x_{N}\right)=\sum_{\boldsymbol{k}} C^{\boldsymbol{k}} \boldsymbol{u}_{0}\left(-\lambda^{\mathbf{k}_{\mathbf{1}}} \mathbf{t}, \mathbf{x}_{\mathbf{2}}-\lambda^{\mathbf{k}_{\mathbf{2}}} \mathbf{t}, \ldots, \mathbf{x}_{\mathbf{N}}-\lambda^{\mathbf{k}_{\mathbf{N}}} \mathbf{t}\right)-\sum_{\boldsymbol{k}: \lambda^{\mathbf{k}_{\mathbf{1}}}<\mathbf{0}} \frac{C^{\boldsymbol{k}} \boldsymbol{A}_{\mathbf{1}}}{\lambda^{\mathbf{k}_{\mathbf{1}}}} \mathbf{y v}
$$

Учитывая (11), имеем

$$
\sum_{\boldsymbol{k}: \lambda^{k_{1}}<0} \frac{1}{\lambda^{k_{1}}} \boldsymbol{C}^{\boldsymbol{k}} \boldsymbol{A}_{1}=\sum_{k_{1}: \lambda^{k_{1}}<0} \frac{1}{\lambda^{k_{1}}} \boldsymbol{C}^{k_{1}} \boldsymbol{A}_{1} .
$$

Поскольку $\boldsymbol{A}_{1}=\boldsymbol{R}_{1} \boldsymbol{\Lambda}_{1} \boldsymbol{\Omega}_{1}$ и $\boldsymbol{C}^{k_{1}}=\boldsymbol{R}_{1} \boldsymbol{D}^{k_{1}} \boldsymbol{\Omega}_{1}$, то $\left(1 / \lambda^{k_{1}}\right) \boldsymbol{C}^{k_{1}} \boldsymbol{A}_{1}=\boldsymbol{C}^{k_{1}}$. Равенство (14) принимает вид

$$
\boldsymbol{u}\left(t, x_{1}=-0, x_{2}, \ldots, x_{N}\right)=\sum_{\boldsymbol{k}} \boldsymbol{C}^{\boldsymbol{k}} \boldsymbol{u}_{0}\left(-\boldsymbol{\lambda}^{k_{1}} t, x_{2}-\boldsymbol{\lambda}^{k_{2}} t, \ldots, x_{N}-\boldsymbol{\lambda}^{k_{N}} t\right)-\sum_{k_{1}: \lambda^{k_{1}}<0} \boldsymbol{C}^{k_{1}} \mathbf{y v} .
$$


Аналогично, для точек $\boldsymbol{x}=\left(x_{1} \geqslant 0, x_{2}, \ldots, x_{N}\right)$, лежащих в правой полуплоскости, с точностью до $\boldsymbol{O}\left(t^{2}\right)$ можем записать

$$
\boldsymbol{u}\left(t, x_{1} \geqslant 0, x_{2}, \ldots, x_{N}\right)=\sum_{\boldsymbol{k}} \boldsymbol{C}^{\boldsymbol{k}} \boldsymbol{u}_{0}\left(\boldsymbol{x}-\boldsymbol{\lambda}^{\boldsymbol{k}} t\right)+\sum_{\boldsymbol{k}: x_{1}<\lambda_{-}^{k_{1}} t} \frac{\boldsymbol{C}^{\boldsymbol{k}} \boldsymbol{A}_{1}}{\lambda^{k_{1}}} \mathbf{y} \mathbf{v}\left(t-\frac{x_{1}}{\lambda^{k_{1}}}, \boldsymbol{x}-\frac{x_{1}}{\lambda^{k_{1}}} \boldsymbol{\lambda}^{\boldsymbol{k}}\right) .
$$

Переходя в (16) к пределу $x_{1} \rightarrow+0$, аналогично (15) получаем

$$
\boldsymbol{u}\left(t, x_{1}=+0, x_{2}, \ldots, x_{N}\right)=\sum_{\boldsymbol{k}} \boldsymbol{C}^{\boldsymbol{k}} \boldsymbol{u}_{0}\left(-\boldsymbol{\lambda}^{k_{1}} t, x_{2}-\boldsymbol{\lambda}^{k_{2}} t, \ldots, x_{N}-\boldsymbol{\lambda}^{k_{N}} t\right)+\sum_{k_{1}: \lambda^{k_{1}}>0} \boldsymbol{C}^{k_{1}} \mathbf{y} \mathbf{v} .
$$

Подставим выражения (15) и (17) в условия сопряжения (3). Получим систему уравнений, которым должен удовлетворять скачок переменных $\mathbf{y v}(t, \boldsymbol{x})$ при переходе через гиперплоскость $\Gamma=\left\{x_{1}=0\right\}:$

$$
\sum_{k_{1}: \lambda^{k_{1}}>0} \boldsymbol{P} \boldsymbol{C}^{k_{1}} \mathbf{y v}-\sum_{k_{1}: \lambda^{k_{1}}<0} \boldsymbol{L} \boldsymbol{C}^{k_{1}} \mathbf{y v}=\boldsymbol{b}^{\boldsymbol{k}}
$$

Здесь

$$
\begin{aligned}
\boldsymbol{b}^{\boldsymbol{k}}=-\sum_{\boldsymbol{k}} \boldsymbol{P} \boldsymbol{C}^{\boldsymbol{k}} \boldsymbol{u}_{0}\left(+0-\boldsymbol{\lambda}^{k_{1}} t, x_{2}-\boldsymbol{\lambda}^{k_{2}} t, \ldots, x_{N}-\boldsymbol{\lambda}^{k_{N}} t\right)- & \\
& -\sum_{\boldsymbol{k}} \boldsymbol{L} \boldsymbol{C}^{\boldsymbol{k}} \boldsymbol{u}_{0}\left(-0-\boldsymbol{\lambda}^{k_{1}} t, x_{2}-\boldsymbol{\lambda}^{k_{2}} t, \ldots, x_{N}-\boldsymbol{\lambda}^{k_{N}} t\right) .
\end{aligned}
$$

Вычтем из уравнения (17) уравнение (15). Получим систему уравнений, которым также должен удовлетворять скачок значений переменных: $\mathbf{y v}(t, \boldsymbol{x})$

$$
\sum_{k_{1}: \lambda^{k_{1}}=0} \boldsymbol{C}^{k_{1}} \mathbf{y v}=\sum_{\boldsymbol{k}: \lambda^{k_{1}}=0} \boldsymbol{C}^{\boldsymbol{k}} \boldsymbol{d}^{\boldsymbol{k}}
$$

здесь

$$
\boldsymbol{d}^{\boldsymbol{k}}=\boldsymbol{u}_{0}\left(+0, x_{2}-\boldsymbol{\lambda}^{k_{2}} t, \ldots, x_{N}-\boldsymbol{\lambda}^{k_{N}} t\right)-\boldsymbol{u}_{0}\left(-0, x_{2}-\boldsymbol{\lambda}^{k_{2}} t, \ldots, x_{N}-\boldsymbol{\lambda}^{k_{N}} t\right) .
$$

Количество линейно независимых уравнений системы (19) равно кратности нулевого собственного числа матрицы $\boldsymbol{A}_{1}$. Умножим равенство (19) на левые собственные векторы-строки матрицы $\boldsymbol{A}_{1}$, соответствующие нулевому собственному числу:

$$
\boldsymbol{l}^{k_{1}} \mathbf{y} \mathbf{v}=\sum_{\boldsymbol{k}: \lambda^{k_{1}}=0} \boldsymbol{l}^{k_{1}} \boldsymbol{C}^{\boldsymbol{k}} \boldsymbol{d}^{\boldsymbol{k}}, \quad k_{1} \text { таково, что } \lambda^{k_{1}}=0 .
$$

Объединим равенства (18) и (20), получим систему линейных алгебраических уравнений, которым должен удовлетворять скачок переменных $\mathbf{y v}(t, \boldsymbol{x})$ при переходе через гиперплоскость $\Gamma=\left\{x_{1}=0\right\}:$

$$
\left\{\begin{array}{l}
\sum_{k_{1}: \lambda^{k_{1}}>0} \boldsymbol{P} \boldsymbol{C}^{k_{1}} \mathbf{y} \mathbf{v}-\sum_{k_{1}: \lambda^{k_{1}}<0} \boldsymbol{L} \boldsymbol{C}^{k_{1}} \mathbf{y} \mathbf{v}=\boldsymbol{b}^{\boldsymbol{k}}, \\
\boldsymbol{l}^{k_{1}} \mathbf{y} \mathbf{v}=\sum_{\boldsymbol{k}: \lambda^{k_{1}}=0} \boldsymbol{l}^{k_{1}} \boldsymbol{C}^{\boldsymbol{k}} \boldsymbol{d}^{\boldsymbol{k}}, \quad k_{1} \text { таково, что } \lambda^{k_{1}}=0 .
\end{array}\right.
$$

Для однозначного решения обобщенной задачи Римана о распаде разрыва с условиями сопряжения на границе необходимо и достаточно, чтобы система уравнений (21) имела единственное решение. Решим систему уравнений (21) и определим значение $\mathbf{y v}(t, \boldsymbol{x})$. Формулы (13) и (16) с полученными зависимостями $\mathbf{y v}(t, \boldsymbol{x})$ дают полное решение обобщенной задачи Римана о распаде разрыва для случая многих пространственных переменных.

Построенное приближение решения обобщенной задачи Римана с дополнительными условиями сопряжения на разрыве в случае одной пространственной переменой является точным. Также это решение будет точным и в случае многих пространственных переменных, если начальные данные заданы линейными функциями по обе стороны гиперплоскости $\Gamma=\left\{x_{1}=0\right\}$. 
Для точек, не лежащих на внутренних или внешних границах, при достаточно малых $t>0$, в соответствии с формулами (13) и (16)

$$
\boldsymbol{u}(t, \boldsymbol{x})=\sum_{\boldsymbol{k}} \boldsymbol{C}^{\boldsymbol{k}} \boldsymbol{u}_{0}\left(\boldsymbol{x}-\boldsymbol{\lambda}^{\boldsymbol{k}} t\right)+\boldsymbol{O}\left(t^{2}\right) .
$$

Обозначим через $\boldsymbol{n}^{\boldsymbol{k}}=\boldsymbol{\lambda}^{\boldsymbol{k}} /\left|\boldsymbol{\lambda}^{\boldsymbol{k}}\right|$ единичный вектор вдоль направления $\boldsymbol{\lambda}^{\boldsymbol{k}}$. Тогда

$$
\frac{\partial \boldsymbol{u}}{\partial t}(t=+0, \boldsymbol{x})=-\sum_{\boldsymbol{k}} \boldsymbol{C}^{k}\left(\boldsymbol{\lambda}^{k} \cdot \frac{\partial \boldsymbol{u}_{0}}{\partial \boldsymbol{n}^{k}}\left(\boldsymbol{x}-0 \cdot \boldsymbol{\lambda}^{k}\right)\right)
$$

здесь

$$
\frac{\partial \boldsymbol{u}}{\partial t}(t=+0, \boldsymbol{x})=\lim _{t \rightarrow+0} \frac{\partial \boldsymbol{u}}{\partial t}(t, \boldsymbol{x}), \quad \frac{\partial \boldsymbol{u}_{0}}{\partial \boldsymbol{n}^{\boldsymbol{k}}}\left(\boldsymbol{x}-0 \cdot \boldsymbol{\lambda}^{\boldsymbol{k}}\right)=\lim _{t \rightarrow+0} \frac{\partial \boldsymbol{u}_{0}}{\partial \boldsymbol{n}^{\boldsymbol{k}}}\left(\boldsymbol{x}-\boldsymbol{\lambda}^{\boldsymbol{k}} t\right) .
$$

Последнее равенство позволяет утверждать, что в указанных точках выполняется равенство

$$
\frac{\partial \boldsymbol{u}}{\partial t}(t, \boldsymbol{x})=-\sum_{\boldsymbol{k}} \boldsymbol{C}^{\boldsymbol{k}}\left(\boldsymbol{\lambda}^{\boldsymbol{k}} \cdot \frac{\partial \boldsymbol{u}}{\partial \boldsymbol{n}^{\boldsymbol{k}}}\left(t, \boldsymbol{x}-0 \cdot \boldsymbol{\lambda}^{\boldsymbol{k}}\right)\right) .
$$

Для точек, лежащих на внутренних границах, введем обозначения

$$
\boldsymbol{u}_{-}(t, \boldsymbol{x})=\boldsymbol{u}\left(t, x_{1}=-0, x_{2}, \ldots, x_{N}\right), \quad \boldsymbol{u}_{+}(t, \boldsymbol{x})=\boldsymbol{u}\left(t, x_{1}=+0, x_{2}, \ldots, x_{N}\right) .
$$

В соответствии с описанным выше алгоритмом, решение задачи Римана по обе стороны границы можно выразить в виде линейной комбинации значений $\boldsymbol{u}_{0}$ в близлежащих точках

$$
\left\{\begin{array}{l}
\boldsymbol{u}_{-}(t, \boldsymbol{x})=\sum_{\boldsymbol{k}} \boldsymbol{S}_{-}^{\boldsymbol{k}}(\boldsymbol{x}) \boldsymbol{u}_{0}\left(\boldsymbol{x}-\boldsymbol{\lambda}^{\boldsymbol{k}} t\right)+\boldsymbol{O}\left(t^{2}\right), \\
\boldsymbol{u}_{+}(t, \boldsymbol{x})=\sum_{\boldsymbol{k}} \boldsymbol{S}_{+}^{\boldsymbol{k}}(\boldsymbol{x}) \boldsymbol{u}_{0}\left(\boldsymbol{x}-\boldsymbol{\lambda}^{\boldsymbol{k}} t\right)+\boldsymbol{O}\left(t^{2}\right) .
\end{array}\right.
$$

$\boldsymbol{S}_{-}^{\boldsymbol{k}}(\boldsymbol{x})$ и $\boldsymbol{S}_{+}^{\boldsymbol{k}}(\boldsymbol{x})$ - матрицы коэффициентов этих линейных комбинаций, получаемые в результате решения задачи Римана. В этих матрицах указана зависимость от точки на внутренней границе, поскольку на разных частях границы могут быть поставлены различные условия сопряжения. Тогда, аналогично (22), в точках внутренних границ выполняются равенства

$$
\left\{\begin{array}{l}
\frac{\partial \boldsymbol{u}_{-}}{\partial t}(t, \boldsymbol{x})=-\sum_{\boldsymbol{k}} \boldsymbol{S}_{-}^{\boldsymbol{k}}(\boldsymbol{x})\left(\lambda^{\boldsymbol{k}} \cdot \frac{\partial \boldsymbol{u}}{\partial \boldsymbol{n}^{\boldsymbol{k}}}\left(t, \boldsymbol{x}-0 \cdot \boldsymbol{\lambda}^{\boldsymbol{k}}\right)\right), \\
\frac{\partial \boldsymbol{u}_{+}}{\partial t}(t, \boldsymbol{x})=-\sum_{\boldsymbol{k}} \boldsymbol{S}_{+}^{\boldsymbol{k}}(\boldsymbol{x})\left(\boldsymbol{\lambda}^{\boldsymbol{k}} \cdot \frac{\partial \boldsymbol{u}}{\partial \boldsymbol{n}^{\boldsymbol{k}}}\left(t, \boldsymbol{x}-0 \cdot \boldsymbol{\lambda}^{\boldsymbol{k}}\right)\right) .
\end{array}\right.
$$

5. Граничные условия. Рассмотрим еще одну задачу, которая далее будет использована при построении вычислительного алгоритма. Необходимо найти в полупространстве $x_{1} \leqslant 0$ решение начально-краевой задачи для систем линейных дифференциальных уравнений первого порядка с постоянными коэффициентами (1), с начальными данными (2). Решение должно быть непрерывным в полупространстве $x_{1} \leqslant 0$ и удовлетворять заданным граничным условиям на гиперплоскости $\Gamma=\left\{x_{1}=0\right\}$ :

$$
\boldsymbol{L u}(t, \boldsymbol{x} \in \Gamma)=\boldsymbol{f}(t, \boldsymbol{x} \in \Gamma) .
$$

Полагаем, что начальные данные удовлетворяют граничным условиям. Эту задачу будем называть обобщенной задачей Римана с граничными условиями.

Пусть $\boldsymbol{u}(t, \boldsymbol{x})$ - решение этой задачи. Доопределим $\boldsymbol{u}(t, \boldsymbol{x})$ нулем для $t<0$ и $t \geqslant 0, x_{1}>0$. Также доопределим нулем $\boldsymbol{u}_{0}(\boldsymbol{x})$ для $x_{1}>0$. Обозначим $\mathbf{y} \mathbf{v}(t, \boldsymbol{x})=-\theta(t) \boldsymbol{u}\left(t, x_{1}=-0, x_{2}, \ldots, x_{N}\right)$.

Как было показано выше, $\boldsymbol{u}(t, \boldsymbol{x})$, рассматриваемая как обобщенная функция из $\boldsymbol{S}^{\prime}$, удовлетворяет уравнению (12). Решение этого уравнения задается формулой (13). Вектор $\mathbf{y v}(t, \boldsymbol{x})$ с точностью $\boldsymbol{O}\left(t^{2}\right)$ удовлетворяет равенствам (15), которые можно переписать в виде

$$
-\sum_{k_{1}: \lambda^{k_{1}} \geqslant 0} \boldsymbol{C}^{k_{1}} \mathbf{y} \mathbf{v}=\sum_{\boldsymbol{k}: \lambda^{k_{1}} \geqslant 0} \boldsymbol{C}^{\boldsymbol{k}} \boldsymbol{u}_{0}\left(-\boldsymbol{\lambda}^{k_{1}} t, x_{2}-\boldsymbol{\lambda}^{k_{2}} t, \ldots, x_{N}-\boldsymbol{\lambda}^{k_{N}} t\right) .
$$


Количество линейно независимых уравнений системы (25) равно количеству линейно независимых собственных векторов матрицы $\boldsymbol{A}_{1}$, соответствующих неотрицательным собственным числам. Умножим равенство (25) на левые собственные векторы-строки матрицы $\boldsymbol{A}_{1}$, соответствующие неотрицательным собственным числам

$$
-\boldsymbol{l}^{k_{1}} \mathbf{y} \mathbf{v}=\sum_{\boldsymbol{k}: \lambda^{k_{1}} \geqslant 0} \boldsymbol{l}^{k_{1}} \boldsymbol{C}^{\boldsymbol{k}} \boldsymbol{u}_{0}\left(-\boldsymbol{\lambda}^{k_{1}} t, x_{2}-\boldsymbol{\lambda}^{k_{2}} t, \ldots, x_{N}-\boldsymbol{\lambda}^{k_{N}} t\right), \quad k_{1} \text { таково, что } \lambda^{k_{1}} \geqslant 0 .
$$

Объединим равенства (26) и (24), получим систему линейных алгебраических уравнений, которым должны удовлетворять значения решения задачи на границе $\Gamma=\left\{x_{1}=0\right\}$ :

$$
\left\{\begin{aligned}
-\boldsymbol{l}^{k_{1}} \mathbf{y} \mathbf{v} & =\sum_{\boldsymbol{k}: \lambda^{k_{1}} \geqslant 0} \boldsymbol{l}^{k_{1}} \boldsymbol{C}^{\boldsymbol{k}} \boldsymbol{u}_{0}\left(-\boldsymbol{\lambda}^{k_{1}} t, x_{2}-\boldsymbol{\lambda}^{k_{2}} t, \ldots, x_{N}-\boldsymbol{\lambda}^{k_{N}} t\right), \quad k_{1} \text { таково, что } \lambda^{k_{1}} \geqslant 0, \\
\boldsymbol{L} \mathbf{y} \mathbf{v} & =-\boldsymbol{f}(t, \boldsymbol{x} \in \Gamma) .
\end{aligned}\right.
$$

Решим систему уравнений (27) и определим значение $\mathbf{y v}(t, \boldsymbol{x})$ при $t>0$ на гиперплоскости $x_{1}=0$. Формулы (13) с полученными зависимостями $\mathbf{y} \mathbf{v}(t, \boldsymbol{x})$ дают полное решение обобщенной задачи Римана с граничными условиями для случая многих пространственных переменных с точностью до $\boldsymbol{O}\left(t^{2}\right)$. Опять же, если начальные данные $\boldsymbol{u}_{0}(\boldsymbol{x})$ являются линейными функциями, то полученное решение является точным решением этой задачи.

В частности, если граничные условия имеют вид

$$
\sum_{k: \lambda_{k_{1}}^{-}<0} C^{k} \mathbf{y v}=\mathbf{0}
$$

что означает, что любые волны проходят через границу, не отражаясь, то такие условия называются «прозрачными». В этом случае, если умножить уравнения граничных условий слева на левые собственные векторы-строки матрицы $\boldsymbol{A}_{1}$, то система уравнений (27) принимает вид

$$
\left\{\begin{aligned}
-\boldsymbol{l}^{k_{1}} \mathbf{y} \mathbf{v} & =\sum_{\boldsymbol{k}: \lambda^{k_{1}} \geqslant 0} \boldsymbol{l}^{k_{1}} \boldsymbol{C}^{\boldsymbol{k}} \boldsymbol{u}_{0}\left(-\boldsymbol{\lambda}^{k_{1}} t, x_{2}-\boldsymbol{\lambda}^{k_{2}} t, \ldots, x_{N}-\boldsymbol{\lambda}^{k_{N}} t\right), & & k_{1} \text { таково, что } \lambda^{k_{1}} \geqslant 0, \\
\boldsymbol{l}^{k_{1}} \mathbf{y} \mathbf{v} & =\mathbf{0}, & & k_{1} \text { таково, что } \lambda^{k_{1}}<0 .
\end{aligned}\right.
$$

Поскольку система уравнений (1) гиперболична и матрица $\boldsymbol{A}_{1}$ имеет полный набор линейно независимых левых собственных векторов, система уравнений (28) совместна и обобщенная задача Римана с прозрачными граничными условиями имеет единственное решение.

Итак, для точек $\boldsymbol{x} \in \Gamma$, лежащих на внешних границах, в соответствии с описанным выше алгоритмом, решение задачи можно выразить в виде линейной комбинации значений $\boldsymbol{u}_{0}$ в близлежащих точках и линейной комбинации компонент вектора $\boldsymbol{f}(t, \boldsymbol{x})$

$$
\boldsymbol{u}=\sum_{\boldsymbol{k}} \boldsymbol{Q}^{\boldsymbol{k}}(\boldsymbol{x}) \boldsymbol{u}_{0}\left(\boldsymbol{x}-\boldsymbol{\lambda}^{\boldsymbol{k}} t\right)+\boldsymbol{P}(\boldsymbol{x}) \boldsymbol{f}(t, \boldsymbol{x})+\boldsymbol{O}\left(t^{2}\right)
$$

где $\boldsymbol{Q}^{\boldsymbol{k}}(\boldsymbol{x})$ и $\boldsymbol{P}(\boldsymbol{x})$ - матрицы коэффициентов этих линейных комбинаций, получаемые в результате решения задачи. Тогда, аналогично (22), в точках внешних границ выполняются равенства

$$
\frac{\partial \boldsymbol{u}}{\partial t}(t, \boldsymbol{x})=-\sum_{\boldsymbol{k}} \boldsymbol{Q}^{\boldsymbol{k}}(\boldsymbol{x})\left(\lambda^{\boldsymbol{k}} \cdot \frac{\partial \boldsymbol{u}}{\partial \boldsymbol{n}^{\boldsymbol{k}}}\left(\boldsymbol{x}-0 \cdot \boldsymbol{\lambda}^{\boldsymbol{k}}\right)\right)+\boldsymbol{P}(\boldsymbol{x}) \frac{\partial \boldsymbol{f}}{\partial t}(t, \boldsymbol{x}) .
$$

6. Распространение упругих волн в блочно-трещиноватой среде. Построение вычислительного алгоритма, основанного на приведенных выше результатах, продемонстрируем на задаче распространения упругих волн в неоднородной блочно-трещиноватой среде. 
6.1. Математическая модель. Систему уравнений, описывающую распространение упругих волн, для случая двух пространственных переменных, следуя [8], можно записать в виде:

$$
\begin{gathered}
\frac{\partial}{\partial t} \sigma_{11}-(\lambda+2 \mu) \frac{\partial}{\partial x_{1}} v_{1}-\lambda \frac{\partial}{\partial x_{2}} v_{2}=0, \\
\frac{\partial}{\partial t} \sigma_{22}-\lambda \frac{\partial}{\partial x_{1}} v_{1}-(\lambda+2 \mu) \frac{\partial}{\partial x_{2}} v_{2}=0, \\
\frac{\partial}{\partial t} \sigma_{12}-\mu \frac{\partial}{\partial x_{1}} v_{2}-\mu \frac{\partial}{\partial x_{2}} v_{1}=0 \\
\rho \frac{\partial}{\partial t} v_{1}-\frac{\partial}{\partial x_{1}} \sigma_{11}-\frac{\partial}{\partial x_{2}} \sigma_{12}=0 \\
\rho \frac{\partial}{\partial t} v_{2}-\frac{\partial}{\partial x_{1}} \sigma_{12}-\frac{\partial}{\partial x_{2}} \sigma_{22}=0
\end{gathered}
$$

где $\lambda$ и $\mu$ коэффициенты Ламе, $\rho$ массовая плотность среды, $\sigma_{11}, \sigma_{22}, \sigma_{12}$ компоненты тензора напряжений, $v_{1}, v_{2}$ компоненты вектора скорости смещений. Введя вектор переменных $\boldsymbol{u}=\left(\sigma_{11}, \sigma_{22}, \sigma_{12}, v_{1}, v_{2}\right)^{T}$ и матрицы

$$
\boldsymbol{A}_{1}=\left(\begin{array}{ccccc}
0 & 0 & 0 & -(\lambda+2 \mu) & 0 \\
0 & 0 & 0 & -\lambda & 0 \\
0 & 0 & 0 & 0 & -\mu \\
-\frac{1}{\rho} & 0 & 0 & 0 & 0 \\
0 & 0 & -\frac{1}{\rho} & 0 & 0
\end{array}\right), \quad \boldsymbol{A}_{2}=\left(\begin{array}{ccccc}
0 & 0 & 0 & 0 & -\lambda \\
0 & 0 & 0 & 0 & -(\lambda+2 \mu) \\
0 & 0 & 0 & -\mu & 0 \\
0 & 0 & 0 & -\frac{1}{\rho} & 0 \\
0 & -\frac{1}{\rho} & 0 & 0 & 0
\end{array}\right)
$$

эту систему уравнений можно записать в виде (1).

Система уравнений (30) является гиперболической, матрицы $\boldsymbol{A}_{1}$ и $\boldsymbol{A}_{2}$ имеют полный набор линейно независимых собственных векторов и представимы в виде (5).

Далее все размерные величины заданы в системе единиц СИ. Поставим для этой системы уравнений следующую задачу. В области $\Omega=\left\{-30<x_{1}<30,-600<x_{2}<0\right\}$ необходимо найти решение начально-краевой задачи для системы уравнений (30). Уравнения (30) должны выполняться всюду в $\Omega$, кроме внутренних границ $\Gamma_{\gamma}, \gamma=1,2$, задаваемых условием $\Gamma_{1}=\left\{x_{1}=\right.$ $-15\}$ и $\Gamma_{2}=\left\{x_{1}=15\right\}$. На этих границах должны выполняться «условия проскальзывания без трения», состоящие в том, что при переходе через эти границы непрерывны нормальные к границам компоненты вектора смещений, нормальные к границе компоненты силы с разных сторон границы равны по величине и противоположно направлены, тангенциальные компоненты сил, действующих по обе стороны границы, равны 0 :

$$
\begin{aligned}
v_{1}\left(t, \boldsymbol{x} \in \Gamma_{\gamma}^{-}\right)-v_{1}\left(t, \boldsymbol{x} \in \Gamma_{\gamma}^{+}\right)=0, & \sigma_{12}\left(t, \boldsymbol{x} \in \Gamma_{\gamma}^{-}\right)=0, \\
\sigma_{11}\left(t, \boldsymbol{x} \in \Gamma_{\gamma}^{-}\right)-\sigma_{11}\left(t, \boldsymbol{x} \in \Gamma_{\gamma}^{+}\right)=0, & \sigma_{12}\left(t, \boldsymbol{x} \in \Gamma_{\gamma}^{+}\right)=0 .
\end{aligned}
$$

На внешних границах $x_{1}=-30, x_{2}=-600, x_{1}=30$ должны выполняться прозрачные граничные условия. На границе $x_{2}=0$ работает источник вибровоздействия, который действует на геологическую среду с усилием $F_{j} \sin \omega t, j=1,2$. Это усилие распределено по границе с плотностью $P_{j}\left(x_{1}\right) \sin \omega t$, так что

$$
\int P_{j} d x_{1}=F_{j}
$$

В соответствии с третьим законом Ньютона во всех точках этой границы должны выполняться условия

$$
\sigma_{12}=-P_{1} \sin \omega t, \quad \sigma_{22}=-P_{2} \sin \omega t
$$

6.2. Численный алгоритм. Внутренними границами область $\Omega$ разбивается на подобласти

$$
\begin{aligned}
& \Omega_{1}=\left\{-30<x_{1}<-15,-600<x_{2}<0\right\} ; \\
& \Omega_{2}=\left\{-15<x_{1}<15,-600<x_{2}<0\right\} ; \\
& \Omega_{3}=\left\{15<x_{1}<30,-600<x_{2}<0\right\} .
\end{aligned}
$$


Построим в каждой из подобластей прямоугольную сетку со сторонами, параллельными осям координат, так, чтобы узлы, лежащие на внутренних границах, совпадали для обеих прилегающих подобластей. Сетку строим равномерной по каждой из координат и $h_{j}, j=1,2$, шаг сетки по соответствующим направлениям. Пусть индекс $p 1$ нумерует узлы сетки в первой подобласти $\Omega_{1}$, индекс $p 2$ нумерует узлы во второй подобласти $\Omega_{2}$ и индекс $p 3$ нумерует узлы сетки в третьей подобласти $\Omega_{3}$. Далее для нумерации узлов сетки в подобласти будем использовать, там где это не вызовет недоразумения, индекс $p$, каждый раз подразумевая, что в каждой подобласти этот индекс пробегает свой набор значений.

Будем полагать, что усилие, создаваемое виброисточником, направлено по вертикали, т. е. $P_{1}\left(x_{1}\right)=0$, а распределение $P_{2}\left(x_{1}\right)$ является кусочно линейной функцией, равной 0 во всех узлах границы $x_{2}=0$, кроме узла с координатами $x_{1}=0, x_{2}=0$. В этом узле $P_{1}$ принимает значение $F_{2} / h_{1}$.

В каждой подобласти построим систему базисных полиномов $H_{p}(\boldsymbol{x})$, каждый из которых равен 1 в узле, соответствующем индексу $p$, равен 0 во всех остальных узлах сетки и в каждой ячейке сетки является билинейной (линейной по каждой переменой) функцией.

Решение в каждой подобласти будем аппроксимировать линейной комбинацией

$$
\boldsymbol{u}(t, \boldsymbol{x})=\sum_{p} H_{p}(\boldsymbol{x}) \boldsymbol{u}^{p}(t)
$$

Как следует из результатов приведенных в предыдущих главах и отраженных в формулах (22), (23) и $(29)$, функции $\boldsymbol{u}^{p}(t)$ удовлетворяют системе линейных обыкновенных дифференциальных уравнений

$$
\frac{\partial \boldsymbol{u}^{p}}{\partial t}(t)=-\sum_{\boldsymbol{k}} \boldsymbol{Q}^{\boldsymbol{k}}\left(\boldsymbol{x}^{p}\right) \sum_{r}\left(\boldsymbol{\lambda}^{\boldsymbol{k}} \cdot \frac{\partial H_{r}}{\partial \boldsymbol{n}^{\boldsymbol{k}}}\left(\boldsymbol{x}^{p}-0 \cdot \boldsymbol{\lambda}^{\boldsymbol{k}}\right)\right) \boldsymbol{u}^{r}(t)+\boldsymbol{P}\left(\boldsymbol{x}^{p}\right) \frac{\partial \boldsymbol{f}}{\partial t}\left(t, \boldsymbol{x}^{p}\right) .
$$

Добавляя к этой системе ОДУ начальные условия

$$
\boldsymbol{u}^{p}(t=0)=\boldsymbol{u}_{0}\left(\boldsymbol{x}^{p}\right)
$$

получаем задачу Коши для системы ОДУ. Решая эту систему тем или иным численным методом, находим значение $\boldsymbol{u}^{p}(t)$ в произвольный момент времени $t$ и по формулам

$$
\boldsymbol{u}(t, \boldsymbol{x})=\sum_{p} H_{p}(\boldsymbol{x}) \boldsymbol{u}^{p}(t)
$$

определяем приближенное решение исходной начально-краевой задачи.

На основании изложенного выше был построен вычислительный алгоритм и реализован в системе Mathlab. Проведенные расчеты показали высокую эффективность этого алгоритма. Выполненные расчеты позволили подтвердить гипотезу, что наличие трещин в геологической породе, в которых соприкасающиеся части могут смещаться друг относительно друга, может приводить к тому, что упругая волна, сгенерированная виброисточником, не рассеивается по полусфере, а распространяется достаточно узким пучком так, что и на существенных глубинах ее плотность энергии остается значимой, чтобы вызвать те или иные физико-химические процессы. Внутренние границы (трещины) ведут себя как стенки волновода. Упругая волна практически не проходит сквозь них, и возмущение источника достигает значительной глубины без рассеяния. Эти и другие результаты вычислительных экспериментов будут подробно изложены в последующих публикациях.

7. Заключение. В работе для гиперболических систем линейных дифференциальных уравнений первого порядка с постоянными коэффициентами, с произвольным количеством пространственных переменных построено приближенное решение обобщенной задачи Римана с условиями сопряжения на разрыве. Также приведено приближенное решение обобщенной задачи Римана с граничными условиями. Для этого построено фундаментальное решение оператора задачи. Это, в свою очередь, позволило свести задачу Римана к решению системы алгебраических уравнений с правой частью, зависящей от значений переменных в начальный момент времени в конечном числе точек. 
На основе этих решений задачи Римана построен и реализован вычислительный алгоритм нахождения решения начально-краевой задачи для гиперболических систем линейных дифференциальных уравнений первого порядка с постоянными коэффициентами. При этом постановка задачи допускает существование внутренних границ, на которых решение может иметь разрывы значений переменных модели и должны выполняться заданные условия, связывающие значения переменных по обе стороны этих границ.

Построенный вычислительный алгоритм был применен для исследования характера распространения упругих волн, сгенерированных периодически действующим виброисточником, в блочно-трещиноватой геологической среде. Существование трещин отражено в модели наличием внутренних границ, на которых выполняются «условия проскальзывания без трения». Проведенные численные эксперименты показали высокую эффективность этого вычислительного алгоритма и его потенциал, как инструмента для исследования практических прикладных задач.

\section{СПИСОК ЛИТЕРАТУРЫ}

1. Владимиров В. С. Уравнения математической физики. - М.: Наука, 1981.

2. Гельфанд И. М., Шилов Г. Е. Некоторые вопросы теории дифференциальных уравнений. - М.: ГИФМЛ, 1958.

3. Гелъфанд И. М., Шилов Г. Е. Обобщенные функции и действия над ними. - М.: ГИФМЛ, 1959.

4. Гельфанд И. М., Шилов Г. Е. Пространства основных и обобщенных функций. - М.: ГИФМЛ, 1958.

5. Куликовский А. Г., Погорелов Н. В., Семенов А. Ю. Математические вопросы численного решения гиперболических систем уравнений. - М.: Физматлит, 2001.

6. Скалько Ю. И. Задача Римана о распаде разрыва в случае многих пространственных переменных// Тр. МФТИ. - 2016. - 8, № 4. - С. 169-182.

7. Скалько Ю. И. Корректные условия на границе, разделяющей подобласти// Компьют. иссл. модел. 2014. - 6, № 3. - C. 347-356.

8. LeVeque R. L. Finite Volume Methods for Hyperbolic Problems. - Cambridge: Cambridge Univ. Press, 2002.

9. Kaser M., Dumbser M. An arbitrary high-order discontinuous Galerkin method for elastic waves on unstructured meshes. I. The two-dimensional isotropic case with external source terms// Geophys. J. Int. - 2006. - 166. - P. 855-877.

Скалько Юрий Иванович

Московский физико-технический институт

(национальный исследовательский университет)

E-mail: skalko@mail.mipt.ru

Гриднев Сергей Юрьевич

Воронежский государственный технический университет

E-mail: gridnev_s_y@rambler.ru 